\title{
The Association of Bite Instability and Comorbidities in Elderly People
}

\author{
Susumu Itakura ${ }^{1,2}$, Masaaki Miyata ${ }^{1}$, Atsushi Kuroda ${ }^{2}$, Manabu Setoguchi $^{2}$, \\ Atsushi Kusumoto ${ }^{2}$, Daisuke Hokonohara ${ }^{2}$ and Mitsuru Ohishi ${ }^{1}$
}

\begin{abstract}
:
Objective The purpose was to evaluate the association between bite instability and comorbidities, comprehensive geriatric evaluations, or disabilities in elderly people.

Methods A dentist examined the oral function, such as the bite stability, number of teeth, and the use of dentures, in 119 patients (93 women, mean age: 86.7 \pm 7.8 ) in 2 nursing homes for the elderly. The association between the oral function and the prevalence of diseases, including hypertension, diabetes mellitus, and dementia, was analyzed.

Results The median number of teeth was $0[0,4]$. The patients were divided into a bite-stable group $(\mathrm{n}=78$, $66 \%)$ and bite-unstable group $(\mathrm{n}=41,34 \%)$. The prevalence of hypertension was significantly higher in the bite-stable group than in the bite-unstable group ( $83 \%$ vs. $63 \%$, respectively; $\mathrm{p}=0.0149$ ), whereas the prevalence of diabetes mellitus was significantly lower in the bite-stable group than in the bite-unstable group $(10 \%$ vs. $27 \%$, respectively; $\mathrm{p}=0.0190)$. The prevalence of a cognitive function decline was significantly lower in the bite-stable group as well (59\% vs. $83 \%, \mathrm{p}=0.0082)$. According to the simplified comprehensive geriatric assessment 7 , the bite-stable group scored significantly higher for instrumental activities of daily living (ADL) than the bite-unstable group (54\% vs. $24 \%$, respectively; $\mathrm{p}=0.0021)$. A multivariate logistic regression analysis demonstrated that bite instability was independently correlated with hypertension, diabetes mellitus, and instrumental activities of daily living.

Conclusion Bite instability was independently associated with a decreased prevalence of hypertension or increased prevalence of diabetes mellitus and low levels of instrumental ADL in the elderly.
\end{abstract}

Key words: bite instability, hypertension, comprehensive geriatric assessment

(Intern Med 57: 1569-1576, 2018)

(DOI: 10.2169/internalmedicine.9830-17)

\section{Introduction}

The aging population is increasing worldwide, and Japan faces an unprecedented, serious, rapid aging of its population (1). Elderly people often develop functional disorders associated with chronic disease or aging (2). There is strong evidence that an increased risk of functional status decline is correlated with cognitive impairment, depression, comorbidities, increased or decreased body mass index (BMI), lower extremity functional limitation, low frequency of social contacts, a low level of physical activity, poor self-perceived health, smoking, and visual impairment $(3,4)$. In addition, a poor dental status is associated with an increased risk of the onset of functional disabilities in elderly Japanese people (5). Oral health is effective for preventing aggravation of diseases, not only in the oral cavity but also generally. Indeed, the number of teeth and the presence of periodontal disease are correlated with the incidence of hypertension (6), cardiovascular disorders (7), cerebrovascular diseases (8), lipid disorders (9), diabetes (10), dementia $(11,12)$, and functional disabilities (5). Furthermore, dental interventions in the oral cavity (13) have been reported to improve refractory hypertension (6), dyslipidemia (9), diabetes (10), and

${ }^{1}$ Department of Cardiovascular Medicine and Hypertension, Graduate School of Medical and Dental Sciences, Kagoshima University, Japan and ${ }^{2}$ Ichihino Memorial Hospital, Japan

Received: July 15, 2017; Accepted: October 15, 2017; Advance Publication by J-STAGE: January 11, 2018

Correspondence to Dr. Masaaki Miyata, miyatam@m3.kufm.kagoshima-u.ac.jp 
pneumonia (14).

However, only a few studies have reported an association between bite stability and comorbidities, and in these reports, the oral status was evaluated by questionnaires rather than by dentists. We hypothesized that oral frailty may be associated with comorbidities seen in elderly persons.

Thus, the primary aim of this study was to evaluate the association of bite stability, as evaluated by a dentist, with the prevalence of comorbidities and disabilities in elderly people, as evaluated by a comprehensive geriatric assessment.

\section{Materials and Methods}

\section{Subjects}

This study evaluated 130 subjects residing in 2 nursing homes for the elderly between April 1, 2015, and July 31, 2016. Full data sets could not be obtained for 11 subjects; therefore, the final analysis included 119 subjects. The study was approved by the Institutional Review Board of Kagoshima University Hospital. Informed consent was obtained from subjects or their legally acceptable representatives.

\section{Measurements of clinical data}

Blood samples were obtained after at least 12 hours of fasting. We measured the serum levels of albumin using a standard technique. In addition, the age, gender, and BMI were determined. For each subject, the blood pressure (BP) was measured in the sitting position by mercury sphygmomanometer, using the first and fifth Korotkoff sounds to identify the systolic and diastolic values, respectively. The cardiothoracic ratio was calculated by chest X-ray. Chest 12lead electrocardiogram was performed to determine the heart rate, atrial fibrillation, left ventricular hypertrophy, ST-T changes, bundle-branch block, and extrasystole.

\section{Measurements of oral status}

An oral examination was performed in the sitting position by a dentist. Bite stability was defined by the ability of a subject to achieve molar occlusion and maximal intercuspal occlusion, regardless of the use of natural teeth or dentures. Subject were divided into two groups - bite-stable and biteunstable groups - according to the results of the bite stability evaluation, which was performed as follows: subjects were positioned with their mandible at rest. In the case of denture wearers, subjects were asked to maintain the rest position of the mandible in the same way as they would while wearing dentures. Using articulating paper, the dentist examined whether or not a subject was able to occlude both molar sections, and each subject was evaluated for the ability to chew while eating.

In addition, the number of the teeth and the presence or absence of dentures were determined, and subjects were classified according to the Eichner index. The Eichner index classification is determined by the presence or absence of occlusal contact in each of the premolar and molar regions, also referred to as supporting zones (15). A maximum of four occlusal support zones (OSZs) can exist, each of which must have at least one tooth in contact with an antagonist tooth to be included. Based on OSZs, subjects were divided into 10 groups as follows: Category A contains the four OSZs as A1 (complete dentition), A2 (missing teeth in one arch), A3 (missing teeth in both arches); Category B contains one to three OSZs or contacts in the anterior area only as B1 (three OSZs), B2 (two OSZs), B3 (one OSZ), and B4 (contacts in the anterior area only); Category $\mathrm{C}$ has no OSZs at all as $\mathrm{C} 1$ (teeth in both arches), C2 (teeth in one arch), and C3 (edentulous).

\section{The evaluation of dementia}

The revised Hasegawa dementia scale (HDS-R) was used to evaluate dementia in the present study. The HDS-R, which consists of nine simple questions with a maximum score of 30, was used to screen for age-associated dementia. A decrease in the cognitive function was defined as a score of $\leq 20$ points on the HDS-R (16).

\section{The evaluation of the comprehensive ability}

The simplified comprehensive geriatric assessment 7 (CGA-7) was performed in this study by a nurse at the nursing home facility. CGA-7 is defined as a multidisciplinary diagnostic and treatment process that identifies medical, psychosocial, and functional limitations of frail, elderly individuals in order to develop a coordinated plan to maximize the overall health with aging. We scored each of seven items with a 1 or 0 as follows: CGA1 (vitality): Can the subject say hello by himself/herself? (1=can; $0=$ cannot); CGA2 (cognitive function; immediately recall): Can the subject repeat the words "cherry tree", "cat", and "train"? ( $1=$ can; $0=$ cannot); CGA3 [instrumental activities of daily living (ADL)]: Can the subject use transportation by himself/herself? (1=can; 0=cannot); CGA4 (cognitive function; late recall): Can the subject recall the words mentioned in CAG2? (1=can; 0=cannot); CGA5 (basic ADL; bathing): Can the subject take a bath without support? (1=can; $0=$ cannot); CGA6 (basic ADL; excretion): Can the subject control urination? ( $1=$ can; $0=$ cannot); CAG7 (emotion): Has the subject felt that he/she is not useful? ( $1=$ no; $0=y e s)$. The total scores ranged from $0-7$, with higher scores representing greater independence (17).

\section{The evaluation of the grip strength and repetitive sa- liva swallowing test}

We considered maintained grip strength as $\geq 26 \mathrm{~kg}$ for men and $\geq 18 \mathrm{~kg}$ for women. For the repetitive saliva swallowing test, a decline in the deglutition function was defined when a subject swallowed two times or less in 30 seconds.

\section{Definition of comorbidities}

We assessed the presence of hypertension, dyslipidemia, 
diabetes mellitus, heart disease, and cerebrovascular disease in each subject. Hypertension was defined per the guidelines of the Japanese Society of Hypertension (JSH) (18) and considered the use of antihypertensive medication and a systolic BP of $\geq 140 \mathrm{mmHg}$ and/or a diastolic BP of $\geq 90$ $\mathrm{mmHg}$. Dyslipidemia was defined per the guidelines of the Japan Atherosclerosis Society (JAS) (19) and considered the use of drugs taken to improve lipid abnormalities, such as low-density-lipoprotein cholesterol $\geq 140 \mathrm{mg} / \mathrm{dL}$, highdensity-lipoprotein cholesterol $<40 \mathrm{mg} / \mathrm{dL}$, or triglyceride $\geq$ $150 \mathrm{mg} / \mathrm{dL}$. Diabetes mellitus was defined per the guidelines of the Japan Diabetes Society (JDS) (20) and considered the use of antidiabetic medications, fasting plasma glucose levels of $\geq 126 \mathrm{mg} / \mathrm{dL}$, casual plasma glucose levels of $\geq 200$ $\mathrm{mg} / \mathrm{dL}$, and a HbA1c of $\geq 6.5 \%$. Heart disease included ischemic heart disease, valvular disease, and cardiomyopathy, and cerebrovascular diseases included cerebral infarction, cerebral hemorrhage, and subarachnoid hemorrhaging. A history of aspiration pneumonitis and fractures was determined based on the medical records.

\section{Statistical analyses}

Data were expressed as the mean \pm standard deviation or median $[25 \%, 75 \%]$. We divided the subjects into bite-stable or bite-unstable groups and compared each parameter investigated between the two groups. Categorical variables were expressed as a percentage and compared between the two groups using the chi-squared test. The unpaired t-test was used for comparisons between different groups of subjects. Student's t-test was used for normally distributed variables, and Wilcoxon's rank sum test was used for variables that were not normally distributed. We used the Shapiro-Wilk test for assessing normality. $\mathrm{p}$ values of $<0.05$ were considered statistically significant. A multivariate logistic regression analysis was performed to evaluate the association between bite instability and comorbidities. All statistical analyses were performed with the JMP Pro software program, version 11 (SAS Institute, Cary, USA) for Windows.

\section{Results}

\section{Clinical characteristics of subjects}

Of the 119 subjects analyzed in the present study, 26 (22\%) were men, and 93 (78\%) were women. The mean age was $86.7 \pm 7.8$ years. Subjects were divided into a bite-stable group $(n=78,66 \%)$ and a bite-unstable group $(n=41,34 \%)$ according to the bite function. The clinical characteristics of subjects are summarized and compared between the bitestable and bite-unstable groups in Table 1. The ratio of women was significantly higher in the bite-stable group than in the bite-unstable group ( $85 \%$ vs. $66 \%$, respectively; $\mathrm{p}=$ 0.0186). There were no significant differences in the age, BMI, or albumin level between the two groups.

\section{Oral characteristics}

The oral status is shown in Table 2. The median number of teeth was $0[0,4]$, and $74 \%(n=88)$ of patients used dentures. Denture use was significantly more frequent in the bite-stable group than in the bite-unstable group (94\% vs. $37 \%$, respectively; $\mathrm{p}<0.0001)$. According to the Eichner index, the number of subjects classified as C2 was significantly lower in the bite-stable group than in the biteunstable group (9\% vs. $24 \%$, respectively; $\mathrm{p}<0.0224$ ). There were no other significant differences in the number of teeth or Eichner index classification between the two groups.

\section{A comparison of the comorbidities between the bite-stable and bite-unstable groups}

The incidence of hypertension was significantly higher in the bite-stable group than in the bite-unstable group $(83 \%$ vs. $63 \%$, respectively; $\mathrm{p}=0.0149$; Figure A). The incidence of diabetes mellitus was significantly lower in the bite-stable group than in the bite-unstable group (10\% vs. $27 \%$, respectively; $\mathrm{p}=0.0190$; Figure B). There were no significant differences in the incidence of dyslipidemia, heart disease, or cerebrovascular disease between the two groups (Table 1).

The cognitive function was evaluated by the HDS-R, and no significant differences were observed in the HDS-R scores between the two groups (Table 1). However, the incidence of an impaired cognitive function, defined as $\leq 20$ points on the HDS-R, was significantly lower in the bitestable group than in the bite-unstable group (59\% vs. $83 \%$, respectively; $\mathrm{p}=0.0082$; Figure $\mathrm{C}$ ).

According to CGA-7, there were no significant differences in the CGA parameters except for instrumental ADL (CGA3) between the two groups. Instrumental ADL (CGA3) was significantly higher in the bite-stable group than in the bite-unstable group ( $54 \%$ vs. $24 \%$, respectively; $\mathrm{p}=0.0021$; Figure D). As shown in Table 1, no significant differences were observed in the grip strength or swallowing function between the two groups. The cardiac indices, including cardiothoracic ratio, heart rate, atrial fibrillation, left ventricular hypertrophy, ST-T changes, bundle-branch block, and extrasystole, did not differ significantly between two groups. In addition, there were no significant differences in the history of aspiration pneumonitis or fractures between the two groups.

In order to adjust for potential confounders, a multivariate logistic regression analysis was performed to analyze the association between bite instability and hypertension, instrumental ADL, a decrease in the cognitive function, or diabetes mellitus. This analysis revealed that bite instability was independently correlated with hypertension, diabetes mellitus, and instrumental ADL (Table 3).

\section{Discussion}

Hypertension is a major cause of cardiovascular disease and other serious health conditions. Koizumi et al. reported 
Table 1. Clinical Characteristics of the Subjects.

\begin{tabular}{|c|c|c|c|c|}
\hline & Total & Bite-stable & Bite-unstable & $\mathrm{p}$ \\
\hline Number of subjects & 119 & $78(66 \%)$ & $41(34 \%)$ & \\
\hline Age, year & $86.7 \pm 7.8$ & $87.6 \pm 7.6$ & $85.0 \pm 8.1$ & 0.0917 \\
\hline Gender (M/F) & $26 / 93$ & $12 / 66$ & $14 / 27$ & 0.0186 \\
\hline Body mass index, $\mathrm{kg} / \mathrm{m}^{2}$ & $22.0 \pm 3.5$ & $22.1 \pm 3.8$ & $21.8 \pm 3.1$ & 0.6261 \\
\hline Albumin, g/dL & $4.0[3.7,4.2]$ & $4.0[3.8,4.2]$ & $3.9[3.7,4.2]$ & 0.5165 \\
\hline \multicolumn{5}{|l|}{ Comorbidity } \\
\hline Hypertension & $91(76.5 \%)$ & $65(83.3 \%)$ & $26(63.4 \%)$ & 0.0149 \\
\hline Dyslipidemia & $41(34.5 \%)$ & $30(38.5 \%)$ & $11(26.8 \%)$ & 0.2045 \\
\hline Diabetes & $19(16.0 \%)$ & $8(10.3 \%)$ & $11(26.8 \%)$ & 0.0190 \\
\hline Heart disease & $28(23.5 \%)$ & $17(21.8 \%)$ & $11(26.8 \%)$ & 0.5384 \\
\hline CVD & $43(36.1 \%)$ & $32(41.0 \%)$ & $11(26.8 \%)$ & 0.1255 \\
\hline \multicolumn{5}{|l|}{ Cognitive function } \\
\hline HDS-R & $18[11,23]$ & $19[11,24]$ & $17[11,20]$ & 0.1542 \\
\hline DCF & $80(67.2 \%)$ & $46(59.0 \%)$ & $34(82.9 \%)$ & 0.0082 \\
\hline \multicolumn{5}{|l|}{ CGA } \\
\hline Total points & $5[3,6]$ & $5[3,6]$ & $5[3,7]$ & 0.4245 \\
\hline CGA1 & $115(96.6 \%)$ & $76(97.4 \%)$ & $39(95.1 \%)$ & 0.5057 \\
\hline CGA2 & $89(74.8 \%)$ & $59(75.6 \%)$ & $30(73.2 \%)$ & 0.7681 \\
\hline CGA3 & $52(43.7 \%)$ & $42(53.9 \%)$ & $10(24.4 \%)$ & 0.0021 \\
\hline CGA4 & $51(42.9 \%)$ & $32(41.0 \%)$ & $19(46.3 \%)$ & 0.5776 \\
\hline CGA5 & $71(59.7 \%)$ & $46(59.0 \%)$ & $25(61.0 \%)$ & 0.8325 \\
\hline CGA6 & $78(65.6 \%)$ & $51(65.4 \%)$ & $27(65.9 \%)$ & 0.9592 \\
\hline CGA7 & $76(63.9 \%)$ & $47(60.3 \%)$ & $29(70.7 \%)$ & 0.2583 \\
\hline \multicolumn{5}{|l|}{ Grip strength } \\
\hline Maintained strength & $5(4.2 \%)$ & $5(6.4 \%)$ & $0(0 \%)$ & 0.0977 \\
\hline \multicolumn{5}{|l|}{ Swallowing function } \\
\hline Good swallowing & $39(32.8 \%)$ & $30(38.5 \%)$ & $9(22.0 \%)$ & 0.0682 \\
\hline \multicolumn{5}{|l|}{ Cardiac function } \\
\hline CTR, $\%$ & $55.9 \pm 6.4$ & $56.4 \pm 6.2$ & $55.0 \pm 6.8$ & 0.2831 \\
\hline Heart rate, bpm & $69.1 \pm 11.0$ & $69.7 \pm 10.5$ & $67.9 \pm 11.9$ & 0.3981 \\
\hline Atrial fibrillation & $4(3.4 \%)$ & $2(2.6 \%)$ & $2(4.9 \%)$ & 0.5057 \\
\hline LVH & $8(6.7 \%)$ & $7(9.0 \%)$ & $1(2.4 \%)$ & 0.5602 \\
\hline ST-T changes & $17(14.3 \%)$ & $12(15.4 \%)$ & $5(12.2 \%)$ & 0.6366 \\
\hline BBB & $18(15.1 \%)$ & $13(16.7 \%)$ & $5(12.2 \%)$ & 0.5177 \\
\hline Extrasystole & $9(7.6 \%)$ & $7(9.0 \%)$ & $2(4.9 \%)$ & 0.4219 \\
\hline \multicolumn{5}{|l|}{ Past history } \\
\hline Aspiration pneumonia & $3(2.5 \%)$ & $2(2.6 \%)$ & $1(2.4 \%)$ & 0.9670 \\
\hline Fracture & $18(15.1 \%)$ & $11(14.1 \%)$ & $7(17.1 \%)$ & 0.6673 \\
\hline
\end{tabular}

Number (\%), average \pm SD, or median [25\%, 75\%].

CVD: cerebrovascular disease, HDS-R: Hasegawa dementia scale revised, DCF: decrease in cognitive function, CGA: comprehensive geriatric assessment, (CGA1: Willingness, CGA2: Cognitive function, CGA3: Instrumental ADL, CGA4: Cognitive function, CGA5: Basic ADL1, CGA6: Basic ADL2, CGA7: State of mind), CTR: cardiothoracic ratio, LVH: left ventricular hypertrophy, BBB: bundle-branch block, ns: not significant

that an impaired oral function as evaluated by questionnaires was associated with uncontrolled hypertension among community-dwelling subjects $\geq 65$ years of age treated for hypertension (21). However, there have been no reports analyzing the correlation between the oral function as evaluated by a dentist and hypertension. Therefore, in the present study, a dentist examined the oral function parameters of bite stability, number of teeth, and use of dentures, and we analyzed the association between the oral function and disease, including the incidence of hypertension, diabetes mellitus, and dementia, in 119 subjects in 2 nursing homes. We demonstrated for the first time that bite instability is associated with the incidence of hypertension and diabetes mellitus and instrumental ADL.

Periodontal disease is a chronic inflammatory condition associated with systemic markers of inflammation and endothelial dysfunction (6). Hypertension is associated with inflammation (22), and several studies have suggested a possible link between periodontal disease and hypertension (23-25). Periodontitis is a low-grade chronic inflammatory disease of the tissues supporting the teeth that may lead to tooth loss (26) and an increase in blood levels of inflam- 
Table 2. Oral Status of the Subjects.

\begin{tabular}{lcccc}
\hline & Total & Bite-stable & Bite-unstable & $\mathrm{p}$ \\
\hline $\begin{array}{l}\text { Number of teeth } \\
\text { Use of denture }\end{array}$ & $0[0,4]$ & $0[0,1.3]$ & $0[0,6.5]$ & 0.5072 \\
Eichner index & 88 & $73(93.6 \%)$ & $15(36.6 \%)$ & $<0.0001$ \\
A1 & $1(0.8 \%)$ & $1(1.3 \%)$ & $0(0 \%)$ & 0.4666 \\
A2 & $4(3.4 \%)$ & $4(5.1 \%)$ & $0(0 \%)$ & 0.1402 \\
A3 & $0(0 \%)$ & $0(0 \%)$ & $0(0 \%)$ & - \\
B1 & $1(0.8 \%)$ & $1(1.3 \%)$ & $0(0 \%)$ & 0.4666 \\
B2 & $1(0.8 \%)$ & $0(0 \%)$ & $1(2.4 \%)$ & 0.1660 \\
B3 & $6(5.0 \%)$ & $4(5.1 \%)$ & $2(4.9 \%)$ & 0.9527 \\
B4 & $9(7.6 \%)$ & $5(6.4 \%)$ & $4(9.8 \%)$ & 0.5118 \\
C1 & $3(2.5 \%)$ & $3(3.9 \%)$ & $0(0 \%)$ & 0.2034 \\
C2 & $17(14.3 \%)$ & $7(9.0 \%)$ & $10(24.4 \%)$ & 0.0224 \\
C3 & $77(64.7 \%)$ & $53(68.0 \%)$ & $24(58.5 \%)$ & 0.3072 \\
\hline
\end{tabular}
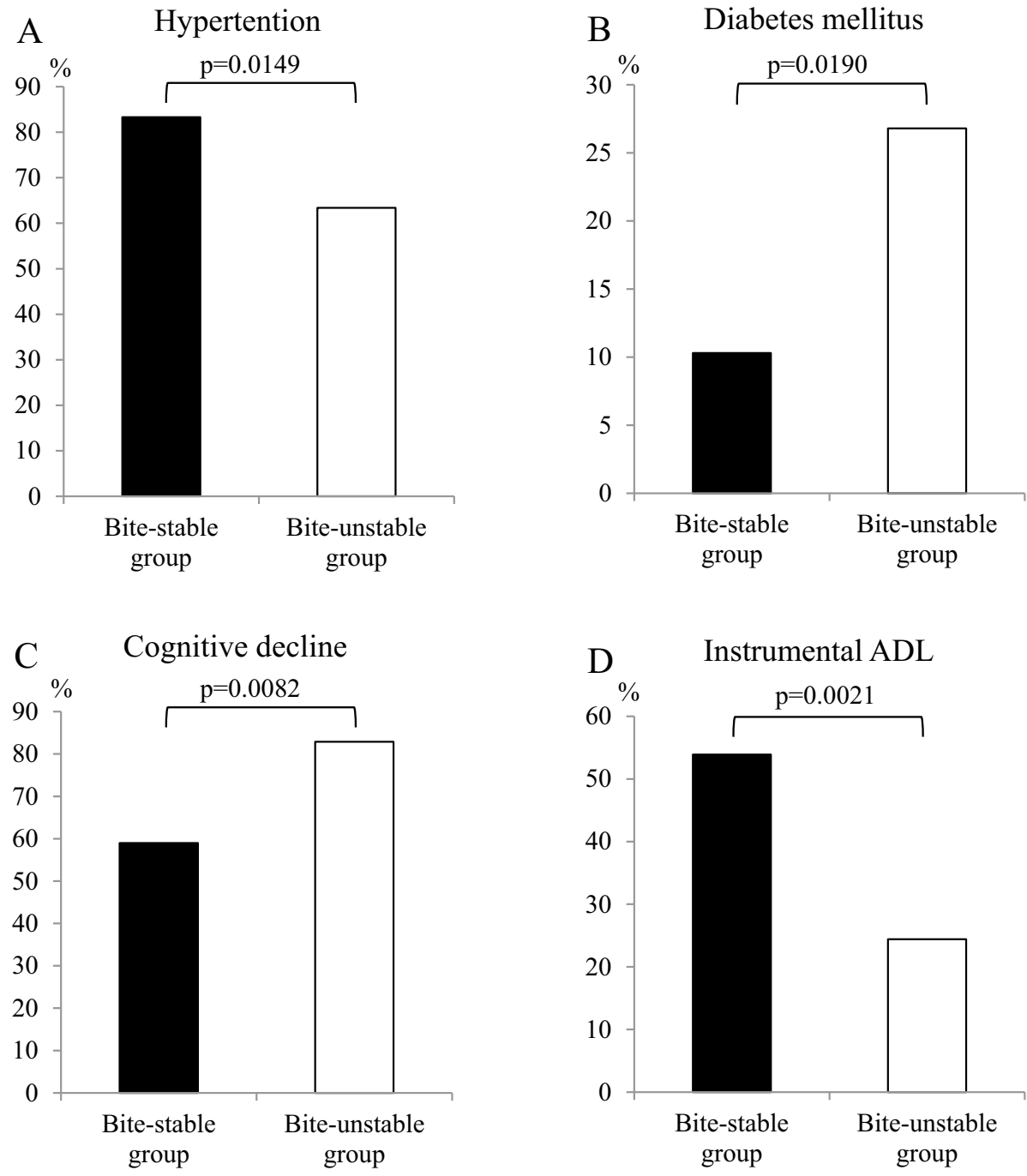

Figure. A comparison of hypertension (A), diabetes mellitus (B), cognitive decline (C), and instrumental ADL (D) between the bite-stable group $(n=78)$ and bite-unstable group $(n=41)$. ADL: activities of daily living

matory markers, including interleukin (IL)-6, C-reactive protein (CRP), and fibrinogen (27-29). Periodontal therapy significantly decreases the levels of CRP, IL-6, and fibrinogen; it also decreases the BP, left ventricular mass, and arterial stiffness, thereby reducing the cardiovascular risk in refractory hypertensive patients (6). Although a strong association 
Table 3. A Multivariate Logistic Regression Analysis between Bite Instability and Hypertension, Diabetes Mellitus, Instrumental Activities of Daily Living, or Decrease in Cognitive Function.

\begin{tabular}{lccc}
\hline & Odds ratio & \multicolumn{1}{c}{$95 \% \mathrm{CI}$} & $\mathrm{p}$ \\
\hline Hypertension & 0.27 & {$[0.10-0.72]$} & 0.0090 \\
Diabetes mellitus & 4.45 & {$[1.43-13.88]$} & 0.0099 \\
Instrumental activities of daily living & 0.28 & {$[0.10-0.82]$} & 0.0196 \\
Decrease in cognitive function & 1.77 & {$[0.57-5.57]$} & 0.3228 \\
\hline CI: confidence interval & & &
\end{tabular}

between periodontal disease and hypertension has been reported, the impact of bite stability on hypertension had not previously been evaluated.

The precise mechanism underlying the association of bite stability with hypertension is unknown. To clarify the possible association of frailty with the prevalence of hypertension and BP control in the elderly, a previous study used the Basic Checklist for Frailty categories to show an independent association between an impaired oral function and uncontrolled hypertension (21). In addition, an independent association was found between difficulty eating hard foods and uncontrolled hypertension. In contrast, our study showed that the incidence of hypertension was significantly higher in the bite-stable group than in the bite-unstable group; we therefore speculated that bite instability might reduce the intake of food and sodium, thereby lowering the BP. Further studies are needed to clarify these mechanisms.

We demonstrated that the incidence of the diabetes mellitus was significantly higher in the bite-unstable group than in the bite-stable group. Recent studies have evaluated the bidirectional relationship between diabetes and periodontal diseases. Strong evidence suggests that diabetes mellitus is associated with an increased prevalence, extent, and severity of gingivitis and periodontitis. As an infectious process with a prominent inflammatory component, periodontal disease can adversely affect the metabolic control of diabetes $(30,31)$. Although the association of periodontal disease with diabetes mellitus has been reported, our study was the first to report the association between bite instability and diabetes mellitus. We speculate that diabetes mellitus aggravates tooth decay and periodontal disease, which may lead to bite instability.

In the present study, we showed that an impaired cognitive function was significantly more common in the biteunstable group than in the bite-stable group. The association between dementia or an impaired cognitive function and the oral hygiene state (32), periodontal disease $(11,33,34)$, number of teeth $(32,33,35,36)$, occlusion, use of dentures $(32,37)$, ability to chew, and the presence or absence of a family dentist (32) has been reported. We therefore speculate that oral disease may increase inflammation, thereby leading to dementia and an impaired cognitive function.

In the present study, the instrumental ADL was significantly higher in the bite-stable group than in the bite- unstable group. Although the precise mechanism underlying the association of instrumental ADL with bite stability is unknown, instrumental ADL is a well-known indicator of the ability to live independently in the community. An inability to perform daily activities independently may affect an individual's ability to maintain good oral hygiene and may restrict their access to necessary dental care. Several crosssectional studies have shown that elderly persons with a functional disability have more untreated caries, a higher prevalence of edentulism, and use dental services less regularly than their counterparts without disabilities (38).

Many studies have reported an association of the number of lost teeth, the residual number of teeth, and periodontal disease with diseases such as hypertension, diabetes mellitus, and dementia. A few studies have analyzed the occlusal state; however, in most studies, the oral status was evaluated by subjective evaluations or questionnaires. In our study, the oral status was evaluated by a dentist.

Several limitations associated with the present study warrant mention. First, the study had a cross-sectional design and did not include a large subject population. Therefore, the causal effect of bite instability on diseases such as hypertension, diabetes mellitus, and dementia could not be determined. We will conduct a longitudinal study to determine the causal effect of bite instability on comorbidities or frailty in a larger number of elderly subjects. Second, we did not evaluate the physical frailty in the present study and were unable to analyze the association between oral frailty and physical frailty.

Regarding the direction of future studies, we should determine the clinical significance of bite stability in cooperation with dentists and hope to examine the preventive effects of chewing rehabilitation using chewing gum and massage of the masseter muscles on the development of diabetes mellitus and the impairment of the cognitive function.

In conclusion, a dentist evaluated the oral function of elderly subjects living in nursing homes, and we found that bite instability was independently correlated with a decreased prevalence of hypertension, an increased prevalence of diabetes mellitus, and low levels of instrumental ADL in elderly individuals. A large, prospective study is needed to confirm these findings.

The authors state that they have no Conflict of Interest (COI). 


\section{Acknowledgement}

We thank the staff at the two nursing homes, Wakouen and Sigetuen, and those at Ichihino Memorial Hospital for their excellent assistance.

\section{References}

1. Murayama H, Nishinaga $M$, Sugawara I, et al. Interactions of household composition and required care level with functional and cognitive status among disabled Japanese elderly living in a suburban apartment complex. Geriatr Gerontol Int 12: 538-546, 2012.

2. Arai H, Ouchi Y, Yokode M, et al.; for the Members of the Subcommittee for Aging. Toward the realization of a better aged society: messages from gerontology and geriatrics. Geriatr Gerontol Int 12: 16-22, 2012.

3. Tas U, Verhagen AP, Bierma-Zeinstra SM, Odding E, Koes BW. Prognostic factors of disability in older people: a systematic review. Br J Gen Pract 57: 319-323, 2007.

4. Stuck AE, Walthert JM, Nikolaus T, Bula CJ, Hohmann C, Beck JC. Risk factors for functional status decline in community-living elderly people: a systematic literature review. Soc Sci Med 48: 445-469, 1999.

5. Aida J, Kondo K, Hirai H, et al. Association between dental status and incident disability in an older Japanese population. J Am Geriatr Soc 60: 338-343, 2012.

6. Vidal F, Cordovil I, Figueredo CM, Fischer RG. Non-surgical periodontal treatment reduces cardiovascular risk in refractory hypertensive patients: a pilot study. J Clin Periodontol 40: 681-687, 2013.

7. Dietrich T, Sharma P, Walter C, Weston P, Beck J. The epidemiological evidence behind the association between periodontitis and incident atherosclerotic cardiovascular disease. J Periodontol 84: S70-S84, 2013.

8. Lafon A, Pereira B, Dufour T, et al. Periodontal disease and stroke: a meta-analysis of cohort studies. Eur J Neurol 21: 11551161, 2014.

9. Acharya A, Bhavsar N, Jadav B, Parikh H. Cardioprotective effect of periodontal therapy in metabolic syndrome: a pilot study in Indian subjects. Metab Syndr Relat Disord 8: 335-341, 2010.

10. Sgolastra F, Severino M, Pietropaoli D, Gatto R, Monaco A. Effectiveness of periodontal treatment to improve metabolic control in patients with chronic periodontitis and type 2 diabetes: a metaanalysis of randomized clinical trials. J Periodontol 84: 958-973, 2013.

11. Sparks Stein P, Steffen MJ, Smith C, et al. Serum antibodies to periodontal pathogens are a risk factor for Alzheimer's disease. Alzheimers Dement 8: 196-203, 2012.

12. Batty GD, Li Q, Huxley R, et al. Oral disease in relation to future risk of dementia and cognitive decline: prospective cohort study based on the Action in Diabetes and Vascular Disease: Preterax and Diamicron Modified-Release Controlled Evaluation (ADVANCE) trial. Eur Psychiatry 28: 49-52, 2013.

13. Yoneyama T, Yoshida M, Ohrui T, et al. Oral care reduces pneumonia in older patients in nursing homes. J Am Geriatr Soc 50: 430-433, 2002.

14. Fukai K, Ouchi A. The spreading movement of prefectural and municipal ordinances for dental and oral health promotion. J Natl Inst Public Health 60: 366-372, 2011.

15. Eichner K. Über eine Gruppeneinteilung des Lückengebisses für die Prothetik. Dtsche Zahnärztl Z 10: 1831-1834, 1955.

16. Imai $Y$, Hasegawa $K$. The revised Hasegawa's dementia scale (HDS-R). Evaluation of its usefulness as a screening test for dementia. Hong Kong J Psychiatry 4: 20-24, 1994.

17. Toba $K$. The guidelines for comprehensive geriatric assessment. Geriatrics 42: 177-180, 2005.

18. Shimamoto K, Ando K, Fujita T, et al.; on behalf of The Japanese
Society of Hypertension Committee for Guidelines for the Management of Hypertension: The Japanese Society of Hypertension Guidelines for the Management of Hypertension (JSH 2014). Hypertens Res 37: 253-392, 2014.

19. Teramoto $T$, Sasaki J, Ishibashi $S$, et al. Diagnostic criteria for dyslipidemia executive summary of the Japan Atherosclerosis Society (JAS). Guidelines for the Diagnosis and Prevention of Atherosclerotic Cardiovascular Diseases in Japan-2012 Version. J Atheroscler Thromb 20: 655-660, 2013.

20. Kuzuya T, Nakagawa S, Satoh J, et al. Committee of the Japan Diabetes Society on the diagnostic criteria of diabetes mellitus: Report of the Committee on the classification and diagnostic criteria of diabetes mellitus. Diabetes Res Clin Pract 55: 65-85, 2002.

21. Koizumi Y, Hamazaki Y, Okuro M, et al. Association between hypertension status and the screening test for frailty in elderly community-dwelling Japanese. Hypertens Res 36: 639-644, 2013.

22. Boos C, Lip G. Elevated high-sensitive C-reactive protein, large arterial stiffness and atherosclerosis: a relationship between inflammation and hypertension? J Hum Hypertens 19: 511-513, 2005.

23. Vidal F, Figueredo CM, Cordovil I, Fischer RG. Higher prevalence of periodontitis in patients with refractory arterial hypertension: a case-control study. Oral Dis 17: 560-563, 2011.

24. Rivas-Tumanyan S, Campos M, Zevallos JC, Joshipura KJ. Periodontal disease, hypertension, and blood pressure among older adults in Puerto Rico. J Periodontol 84: 203-211, 2013.

25. Desvarieux M, Demmer RT, Jacobs DR, et al. Periodontal bacteria and hypertension: the oral infections and vascular disease epidemiology study (INVEST). J Hypertens 28: 1413-1421, 2010.

26. Moutsopoulos NM, Madianos PN. Low-grade inflammation in chronic infectious diseases: paradigm of periodontal infections. Ann N Y Acad Sci 1088: 251-264, 2006.

27. Loos BG, Craandijk J, Hoek FJ, Wertheim-van Dillen PM, van der Velden U. Elevation of systemic markers related to cardiovascular diseases in the peripheral blood of periodontitis patients. J Periodontol 71: 1528-1534, 2000.

28. Paraskevas S, Huizinga JD, Loos BG. A systematic review and meta-analyses on $\mathrm{C}$-reactive protein in relation to periodontitis. $\mathrm{J}$ Clin Periodontol 35: 277-290, 2008.

29. Vidal F, Figueredo CM, Cordovil I, Fischer RG. Periodontal therapy reduces plasma levels of interleukin-6, C-reactive protein, and fibrinogen in patients with severe periodontitis and refractory arterial hypertension. J Periodontol 80: 786-791, 2009.

30. Taylor GW. Bidirectional interrelationships between diabetes and periodontal diseases: an epidemiologic perspective. Ann Periodontol 6: $99-112,2001$.

31. Mealey BL. Periodontal disease and diabetes. A two-way street. J Am Dent Assoc 137: 26s-31s, 2006.

32. Yamamoto T, Kondo K, Hirai H, Nakade M, Aida J, Hirata Y. Association between self-reported dental health status and onset of dementia: a 4-year prospective cohort study of older Japanese adults from the Aichi Gerontological Evaluation Study (AGES) Project. Psychosom Med 74: 241-248, 2012.

33. Kaye EK, Valencia A, Baba N, Spiro A, Dietrich T, Garcia RI. Tooth loss and periodontal disease predict poor cognitive function in older men. J Am Geriatr Soc 58: 713-718, 2010.

34. Stewart R, Weyant RJ, Garcia ME, et al. Adverse oral health and cognitive decline: the health, aging and body composition study. J Am Geriatr Soc 61: 177-184, 2013.

35. Kondo K, Niino M, Shido K. A case-control study of Alzheimer's disease in Japan significance of life-styles. Dementia 5: 314-326, 1994.

36. Batty GD, Li Q, Huxley R, et al. Oral disease in relation to future risk of dementia and cognitive decline: prospective cohort study based on the Action in Diabetes and Vascular Disease: Preterax and Diamicron Modified-Release Controlled Evaluation (ADVANCE) trial. Eur Psychiatry 28: 49-52, 2013. 
37. Paganini-Hill A, White SC, Atchison KA. Dentition, dental health habits, and dementia: the Leisure World Cohort Study. J Am Geriatr Soc 60: 1556-1563, 2012.

38. Wu B, Plassman BL, Liang J, Wei L. Cognitive function and dental care utilization among communitydwelling older adults. Am J Public Health 97: 2216-2221, 2007.
The Internal Medicine is an Open Access article distributed under the Creative Commons Attribution-NonCommercial-NoDerivatives 4.0 International License. To view the details of this license, please visit (https://creativecommons.org/licenses/ by-nc-nd/4.0/).

(C) 2018 The Japanese Society of Internal Medicine Intern Med 57: 1569-1576, 2018 